\title{
SÍNDROME DE IRIS FLÁCCIDO POR TAMSULOSINA. ESTUDIO PROSPECTIVO FRENTE A GRUPO CONTROL
}

\section{FLOPPY-IRIS SYNDROME ASSOCIATED WITH TAMSULOSIN. A PROSPECTIVE CASE-CONTROL STUDY}

\author{
BIDAGUREN A ${ }^{1}$, IRIGOYEN $C^{1}$, MENDICUTE J ${ }^{2}$, GUTIÉRREZ E ${ }^{3}$, GIBELALDE A ${ }^{1}$, ÚBEDA M ${ }^{2}$
}

\section{RESUMEN}

Objetivos: Los objetivos de este estudio son: determinar la incidencia real del síndrome del iris fláccido intraoperatorio asociado al tratamiento con tamsulosina y analizar las diferentes tasas de complicaciones tanto intra como postoperatorias frente a un grupo control. Los objetivos secundarios son: describir las alteraciones pupilares asociadas a la toma de tamsulosina y cuantificar la pérdida endotelial de la cirugía de catarata.

Método: Estudio prospectivo que incluye 38 ojos de 38 pacientes divididos en dos grupos. El grupo 1 (casos) compuesto por 19 ojos de 19 pacientes varones en tratamiento con tamsulosina y el grupo 2 (controles) compuesto por 19 ojos de 19 varones sin tratamiento con tamsulosina. En todos los casos, se trata de pacientes consecutivos que acuden a nuestro hospital para ser intervenidos de catarata.

Resultados: La incidencia de iris fláccido en nuestra serie se limita a dos pacientes $(10 \%)$ que presentaron la tríada completa, 9 pacientes (47\%) presentaron 2 de los 3 signos, y solo dos pacientes $(10 \%)$ presentaron bamboleo del iris aislado. Ninguno de los pacientes del grupo control presentó
Objective: The main objectives of this study were: To assess the incidence of the intraoperative floppyiris syndrome associated with tamsulosin and to analyse the incidence of intraoperative and postoperative complications as compared to a control group. Secondary objectives were: to describe the pupillary modifications associated with tamsulosin and to quantify the endothelial cell loss.

Methods: A prospective review of 38 eyes of 38 patients was performed. Patients were assigned to two different groups. Group 1 (cases) included 19 eyes of 19 male patients taking tamsulosin, and group 2 (controls) included 19 eyes of 19 male patients not taking tamsulosin.

Results: Only two patients (10\%) of our study had the complete triad seen in floppy-iris syndrome; 9 patients (47\%) showed 2 of the 3 main features of the syndrome and only 2 patients showed iris billowing during phacoemulsification. None of the patients in group 2 showed any of the characteristic intraoperative features. The complication rate was similar in both groups. Conclusions: Intraoperative floppy-iris syndrome occurred in $67 \%$ of the patients treated with tamsu-

\footnotetext{
Recibido: 31/5/06. Aceptado: 24/5/07.

Hospital Donostia. San Sebastián. Guipúzcoa. España.

1 Licenciado en Medicina.

2 Doctor en Medicina.

3 Óptico-Optometrista.

Correspondencia:

Aritz Bidaguren

Paseo Dr. Marañón, $14,3 .^{\circ} \mathrm{C}$

20009 San Sebastián (Guipúzcoa)

España

E-mail: aritzbidaguren@kaixo.com
} 
característica alguna de este síndrome. La incidencia de complicaciones fue similar en ambos grupos. Conclusiones: Como conclusión mencionar que el síndrome IFIS se presenta en el 67\% de los pacientes en tratamiento con tamsulosina. El único efecto secundario postoperatorio a destacar ha sido la observación de una menor reactividad pupilar en los pacientes tratados con tamsulosina, circunstancia no descrita en la literatura.

Palabras clave: Síndrome de Iris fláccido, tamsulosina, Catarata, $\alpha$-bloqueantes, ondulaciones iris. losin. The only postoperative secondary effect was a lower pupil reactivity in patients taking tamsulosin (Arch Soc Esp Oftalmol 2007; 82: 349-354).

Key words: Floppy-iris syndrome, tamsulosin, cataract, alpha blockers, iris billowing.

\section{INTRODUCCIÓN}

Una inadecuada dilatación pupilar o las modificaciones pupilares observadas intraoperatoriamente pueden comprometer la cirugía de catarata e incrementar el riesgo de complicaciones (1). El síndrome de iris fláccido intraoperatorio (Intraoperative floppy iris syndrome: IFIS) es una situación intraoperatoria recientemente descrita en relación con las modificaciones pupilares observadas en pacientes que toman tamsulosina (2). El IFIS está caracterizado por la presencia de tres signos intraoperatorios: un estroma iridiano fláccido que se ondula en condiciones de fluídica habituales, tendencia del iris a prolapsarse a través de las incisiones de facoemulsificación y paracentesis adecuadamente construidas, y contracción pupilar intraoperatoria progresiva, independiente de las pautas farmacológicas habituales para lograr la midriasis (2). Se ha descubierto que una de las causas desencadenantes del mencionado síndrome es la toma por parte de los pacientes de tamsulosina (2-7), un bloqueante de los receptores adrenérgicos alfa-1 (8) utilizado para el tratamiento de la hiperplasia benigna de próstata (9-12).

El mencionado síndrome ha sido descrito recientemente y por lo tanto no existe información suficiente sobre su prevalencia real. Chang y Campbell (2) refieren que la prevalencia de pacientes en tratamiento con tamsulosina es del $3 \%$ en su grupo poblacional de pacientes operados de catarata. Los mismos autores (2) refieren que el síndrome se presenta en más del $60 \%$ de los pacientes en tratamiento con la mencionada medicación. Aún es preciso definir la duración del tratamiento con tamsulosina que es necesaria para desarrollar el síndrome, así como el impacto del desarrollo del IFIS sobre los resultados de la cirugía de catarata.

Con tal propósito se inició el presente estudio cuyos objetivos fueron: determinar la incidencia real del síndrome del iris fláccido en pacientes en tratamiento con tamsulosina y analizar la incidencia de complicaciones tanto intra como postoperatorias en dos grupos de pacientes de características similares que tan solo se diferencian en función de la toma o no de tamsulosina. Los objetivos secundarios fueron: describir las alteraciones pupilares asociadas a la toma de tamsulosina y cuantificar la pérdida endotelial que la cirugía de catarata tiene en estos dos grupos de pacientes.

\section{SUJETOS, MATERIAL Y MÉTODO}

Se realiza un estudio prospectivo que incluye 38 ojos de 38 pacientes diferentes divididos en dos grupos. El grupo 1 (casos) compuesto por 19 ojos de 19 pacientes varones en tratamiento con tamsulosina y el grupo 2 (controles) compuesto por 19 ojos de 19 varones que no estaban en tratamiento con tamsulosina. En todos los casos, se trataba de pacientes que acudieron a nuestro hospital para ser intervenidos de catarata a los que se asigna a estos dos grupos de acuerdo a los siguientes criterios: los pacientes en tratamiento con tamsulosina que acudieron para ser intervenidos de catarata fueron incluidos consecutivamente en el grupo 1, mientras que todo paciente varón que acudiera inmediatamente después de un caso fue introducido en el grupo 2 (control). Se excluyeron, en ambos grupos, todos aquellos pacientes que presentaban alguna patología oftalmológica que pudiera comprometer 
la cirugía como pueden ser los episodios de traumatismos previos o la presencia de desinserciones zonulares. Todos los pacientes fueron intervenidos por un único cirujano en el período comprendido entre noviembre de 2005 y mayo de 2006.

Previo a la cirugía, y en todos los pacientes, se realizó una anamnesis sobre las patologías oftalmológicas y sistémicas que pudieran alterar los resultados de nuestro estudio así como un detallado examen oftalmológico que incluyó lo siguiente: agudeza visual preoperatoria, determinación del grado de catarata, color del iris, biometría y medida de la profundidad de cámara anterior mediante IOL Master (Zeiss, Jena, Germany) pupilometría (CSO Eye TOP 6.4, CSO, Italia) en condiciones fotópicas, mesópicas, escotópicas y tras dilatación con tropicamida y recuento endotelial. Intraoperatoriamente se estudió la pauta de midriasis, el tipo de anestesia empleado, el tiempo de ultrasonidos durante la facoemulsificación, la presencia de los tres signos que componen el síndrome del iris fláccido así como las complicaciones quirúrgicas y la necesidad de procedimientos adicionales durante la cirugía.

Se realizaron visitas al día siguiente, a la semana y al mes, en la cual se valoró la agudeza visual final, el recuento endotelial y se realizó una nueva pupilometría en condiciones fotópicas, mesópicas, escotópicas y tras dilatación con tropicamida.

\section{RESULTADOS}

Ambos grupos de pacientes fueron comparables en relación a la edad $(\mathrm{p}=0,42)$ con una edad media de 76,7 DE 5,81 años (media y desviación estándar) en el grupo 1 y de 75,3 DE 4,77 años en el grupo 2. De igual modo, los dos grupos resultaron comparables en biometría y profundidad de cámara anterior, con una longitud axial media de 23,5 DE 0,4 mm en el grupo 1 y 23,41 DE 0,53 $\mathrm{mm}$ en el grupo 2, diferencias que resultaron estadísticamente no significativas $(\mathrm{p}=0,7)$. Las agudezas visuales preoperatorias fueron de 0,37 en el grupo 1 y 0,37 en el grupo 2 respectivamente. Las diferencias entre el tamaño preoperatorio de las pupilas realizado mediante pupilómetro (CSO Eye TOP 6.4) (CSO Ophthalmic, Firenze, Italia) mostró un diámetro pupilar comparable en ambos grupos mostrando las siguientes medidas (tabla I): 1) Condiciones fotópicas: $3,11 \mathrm{DE} 0,58$ y $3,43 \mathrm{DE} 0,57 \mathrm{~mm}$ respectivamente $(\mathrm{p}=0,15)$; 2) Mesópicas: 3,71 DE 0,79 y 3,9
Tabla I. Diámetro pupilar preoperatorio medio en milímetros

\begin{tabular}{llr}
\hline & Grupo 1 & \multicolumn{1}{c}{ Grupo 2 } \\
\hline Fotópica & $3,11 \pm 0,58$ & $3,41 \pm 0,57$ \\
Mesópica & $3,71 \pm 0,79$ & $3,9 \pm 0,52$ \\
Escotópica & $3,95 \pm 0,8$ & $4,1 \pm 0,59$ \\
Tras tropicamida & $6,42 \pm 0,74$ & $6,18 \pm 0,86$ \\
\hline \hline
\end{tabular}

Media en milímetros \pm desviación estandar.

DE 0,52 mm ( $\mathrm{p}=0,39)$; 3) Escotópicas: 3,95 DE 0,8 y 4,10 DE $0,59 \mathrm{~mm}(\mathrm{p}=0,53)$; y 4) Tras dilatación durante 20 minutos con tropicamida: 6,42 DE 0,74 y $6,18 \mathrm{DE} 0,86 \mathrm{~mm}(\mathrm{p}=0,38)$ respectivamente. Tampoco hubo diferencias preoperatorias $(p=0,82)$ entre el recuento endotelial realizado previo a la cirugía en ambos grupos, siendo este de 2267 DE 285 células $/ \mathrm{mm}^{2}$ en el grupo 1 y 2418 DE 417 células $/ \mathrm{mm}^{2}$ en el grupo 2.

La pauta de midriasis se realizó, en todos los casos, de forma rutinaria mediante homatropina (Colircusí Homatropina ${ }^{\circledR}$. Alcon Cusí, SA. El Masnou. Barcelona. España) y fenilefrina (Colircusí Fenilefrina ${ }^{\circledR}$. Alcon Cusí, SA. El Masnou. Barcelona. España), sin necesidad de realizar procedimientos adicionales por insuficiente midriasis intraoperatoria. Todos los pacientes fueron intervenidos con anestesia tópica. Con respecto a la presencia de complicaciones intraoperatorias, éstas se limitaron a un paciente con catarata intumescente del grupo control (Grupo 2) que presentó un rasgado de la rexis anterior realizada bajo azul tripan $0,1 \%$ (Vision Blue) (Signo de la «bandera argentina») y que se resolvió favorablemente con la implantación de la lente intraocular en sulcus y sin necesidad de vitrectomía por ausencia de vitreorragia y a un paciente del grupo 1 (tamsulosina) que presentó una desinserción zonular y vitreorragia y que precisó de vitrectomía anterior. El tiempo de ultrasonidos empleado durante la facoemulsificación fue de 66 DE 5 y 58 DE 6 segundos de media $(p=0,34)$ en los grupos 1 y 2 respectivamente. No se realizó ninguna maniobra intraoperatoria adicional para poder conseguir una adecuada midriasis en ninguno de los pacientes.

En relación a la incidencia del síndrome de iris fláccido definido por la tríada: iris fláccido que se ondula en condiciones de fluídica habituales, tendencia del iris a prolapsarse a través de incisiones correctamente tunelizadas y tendencia a la miosis intraoperatoria progresiva con las pautas de midria- 
sis convencionales, cabe destacar lo siguiente: solo dos pacientes (10\%) presentaron la tríada completa, mientras que nueve pacientes $(47 \%)$ presentaron dos de los 3 signos y solo 2 pacientes (10\%) presentaron bamboleo de iris aislado sin presencia de ninguno de los otros signos. De este modo, la presencia de algún signo definitorio del síndrome del iris fláccido se limitó al $67 \%$ de los pacientes en tratamiento con tamsulosina mientras que ninguno de los pacientes del grupo control manifestó característica alguna de este síndrome.

Tras la facoemulsificación, los pacientes siguieron una pauta de revisiones similar en ambos grupos obteniendo medidas de presión intraocular (PIO) media similar tanto en el postoperatorio inmediato como a la semana de la intervención. La PIO el primer día postoperatorio fue de 16,37 DE 3,95 y 17,37 DE 4,5 mmHg respectivamente y a la semana de la cirugía la PIO media fue de 13,42 DE 2,55 y 14,05 DE 3,34 $\mathrm{mmHg}$ respectivamente, sin que existiera ningún caso de hipertensión postoperatoria.

Con respecto a la modificación postoperatoria del diámetro pupilar (tabla II), en el grupo 1 se evidenció una tendencia a la disminución del diámetro postoperatorio de la pupila tanto en condiciones fotópicas, mesópicas, escotópicas y tras dilatación con tropicamida con los siguientes tamaños de pupila medio: 2,77 DE 0,52; 3,33 DE 0,77; 3,55 DE 0,83 y $5,0 \mathrm{DE} 0,94 \mathrm{~mm}$ y una reducción porcentual respecto de la pupila preoperatoria del 11\%, 10\%, $10 \%$ y $22 \%$ respectivamente. Por el contrario, la pupilometría de los pacientes del grupo 2 mostró una menor tendencia hacia la disminución del diámetro pupilar postoperatorio tanto en condiciones fotópicas, mesópicas, escotópicas y tras dilatación con tropicamida, con las siguientes medidas: 3,17 DE 0,4; 3,72 DE 0,57; 4,03 DE 0,75 y 5,54 DE $0,8 \mathrm{~mm}$ y una disminución porcentual de la pupila del $8,9 \%, 5 \%, 0,02 \%$ y $10 \%$ respectivamente. La comparación de las medias de las diferencias entre las pupilas pre y postoperatorias en ambos grupos utilizando una prueba $\mathrm{T}$ para muestras independientes es menor en el grupo de pacientes en tratamiento con tamsulosina, siendo estas diferencias estadísticamente significativas para las pupilas mesópicas, escotópicas y tras dilatación durante 20 minutos con tropicamida con valores de $\mathrm{p}$ a dos colas de 0,$05 ; 0,07$ y $<0,001$ respectivamente.

Como ocurre habitualmente tras la facoemulsificación, en ambos grupos de pacientes se encontró una pérdida de celularidad endotelial del $13 \%$ y $24 \%$ respectivamente, no habiendo diferencias estadísticamente significativas entre ambos grupos.

\section{DISCUSIÓN}

Desde la descripción del síndrome de iris fláccido intraoperatorio (IFIS) por uso de tamsulosina por Chang y Campbell en el año 2005 (2), y debido a un mayor riesgo de complicaciones intraoperatorias, tal circunstancia ha despertado el interés de los cirujanos de cataratas. Por ello, registrar adecuadamente y de forma preoperatoria a los pacientes que presentando hiperplasia benigna de próstata (HBP) estén en tratamiento con tamsulosina, permitirá planificar mejor la cirugía de catarata, evitando complicaciones derivadas de dificultades técnicas inesperadas $(2,13)$.

En la serie inicial de los mencionados autores (2) encontraron que un $3 \%$ de sus pacientes operados de catarata estaban en tratamiento con tamsulosina y que el IFIS se desarrollaba en el 67-100\% de dichos pacientes. Del mismo modo, un alto porcentaje (31\%-48\%) de ellos precisó de alguna maniobra pupilar para lograr una adecuada midriasis durante la facoemulsificación. Sin embargo, otros autores (15) en Gran Bretaña encontraron una menor incidencia asociada al tratamiento con tamsulosina en su población de estudio $(0,71 \%)$, y ni el

Tabla II. Comparación de los diámetros pupilares pre y postoperatorios en milímetros

\begin{tabular}{lcccccrr}
\hline & $\begin{array}{c}\text { Media } \\
\text { preop. }\end{array}$ & $\begin{array}{c}\text { Grupo } 1 \\
\text { Media } \\
\text { postop. }\end{array}$ & $\begin{array}{l}\text { Diferencia } \\
\text { de medias }\end{array}$ & $\begin{array}{l}\text { Media } \\
\text { preop. }\end{array}$ & $\begin{array}{c}\text { Grupo } 2 \\
\text { Media } \\
\text { postop. }\end{array}$ & \multicolumn{1}{c}{$\begin{array}{l}\text { Diferencia } \\
\text { de medias }\end{array}$} & Valor $p$ \\
\hline Fotópica & $3,11 \pm 0,58$ & $2,77 \pm 0,52$ & $0,44 \pm 0,1$ & $3,4 \pm 0,57$ & $3,17 \pm 0,4$ & $0,24 \pm 0,13$ & 0,23 \\
Mesópica & $3,71 \pm 0,79$ & $3,33 \pm 0,77$ & $0,48 \pm 0,1$ & $3,9 \pm 0,52$ & $3,72 \pm 0,57$ & $0,15 \pm 0,13$ & 0,05 \\
Escotópica & $3,95 \pm 0,8$ & $3,55 \pm 0,83$ & $0,48 \pm 0,17$ & $4,1 \pm 0,59$ & $4,03 \pm 0,75$ & $0,04 \pm 0,16$ & 0,07 \\
Tropicamida & $6,42 \pm 0,74$ & $5 \pm 0,94$ & $1,52 \pm 0,2$ & $6,18 \pm 0,86$ & $5,54 \pm 0,8$ & $0,61 \pm 0,09$ & $<0,01$ \\
\hline \hline
\end{tabular}

Medias en milímetros \pm desviación estándar; valor p de significación estadística para la comparación de las medias de las diferencias entre las pupilas pre y postoperatorias en ambos grupos mediante la prueba $\mathrm{T}$ para muestras independientes. 
desarrollo del IFIS fue tan frecuente ni la necesidad de practicar maniobras pupilares tan alta como en la serie original. En nuestra serie, la incidencia de IFIS en tomadores de tamsulosina ha sido del $67 \%$, aunque tan solo un $10 \%$ de los pacientes presentaban el cuadro clínico completo. Por otro lado, y al analizar cada uno de los signos intraoperatorios que definen el IFIS, cabe destacar que las ondulaciones iridianas han sido el signo más frecuente en nuestra serie ( $47 \%$ de los casos). Igualmente, hemos podido comprobar que la severidad del IFIS es variable y que no necesariamente se manifiesta con la tríada clínica completa.

El tratamiento con tamsulosina o la presencia de IFIS no son, en principio, contraindicación para la facoemulsificación. Sin embargo, la posibilidad de un mayor número de complicaciones derivadas de la dificultad técnica que presenta la cirugía de catarata en estos pacientes (1) hace necesarias una serie de medidas preventivas previas a la cirugía. Así, además de una correcta anamnesis y ante el antecedente de la toma de tamsulosina por parte de los pacientes, conviene disponer de retractores de iris y de viscoelásticos supercohesivos para aquellos casos en los que las maniobras midriáticas habituales no eviten la aparición de una miosis intraoperatoria que nos imposibilite continuar con la facoemulsificación. De este modo, y a pesar de que nosotros no hayamos encontrado una mayor incidencia de complicaciones en el grupo de pacientes en tratamiento con tamsulosina, entendemos que la presencia del IFIS dificulta notablemente la facoemulsificación en estos ojos. El mayor tiempo de ultrasonidos empleado en el grupo de pacientes en tratamiento con tamsulosina, y en especial en aquellos casos que presentaron alguna de las características definitorias del IFIS, pone de manifiesto esta mayor dificultad técnica y aunque en esta serie no se haya encontrado una pérdida de celularidad endotelial mayor en el grupo con tamsulosina, una muestra de mayor tamaño podría mostrar esa pérdida de células endoteliales generada por el mayor consumo de energía (14). Por otro lado, y como se ha mencionado previamente, la severidad del síndrome no siempre es la misma ya que, tanto la dificultad quirúrgica como el riesgo de complicaciones es mayor en los casos que presentan la tríada clínica completa. De cualquier modo, entendemos que la facoemulsificación en pacientes con IFIS es segura siempre que se realice por cirujanos expertos y se hayan tomado todas las precauciones necesarias.
Debido a la reciente descripción de este síndrome, aún no se ha determinado la relación entre la duración del tratamiento con tamsulosina y el desarrollo del IFIS. Sin embargo, la hipótesis patogénica que sostiene que el síndrome se debe a una atrofia por desuso del músculo dilatador del iris (2) sugiere que un tiempo de tratamiento más largo aumentaría el riesgo de desarrollar IFIS y su severidad. Por otro lado, queda aún por determinar la utilidad o no de suspender la medicación con anterioridad a la cirugía. Debido a que la vida media de la tamsulosina en sangre es de 48-72 horas, parece que suspender la medicación entre 4 y 7 días previos a la cirugía podría ser de ayuda aunque no evite la aparición del IFIS (2). Así, la mayoría de los autores recomienda suspender la medicación de 1 a 2 semanas previas a la cirugía, aunque su utilidad esté todavía por demostrar $(2,7)$.

Una menor midriasis preoperatoria es común a todos los pacientes que toman algún tipo de alfabloqueante (2). Nosotros hemos visto esta tendencia en nuestros pacientes del grupo de tamsulosina aunque las diferencias con el grupo control no han sido estadísticamente significativas. Sin embargo, se ha detectado una tendencia hacia la miosis postquirúrgica en pacientes en tratamiento con tamsulosina mayor que en los pacientes del grupo control. Estas diferencias han resultado ser estadísticamente significativas excepto en condiciones fotópicas y tal vez pudieran deberse a la mayor manipulación instrumental sobre el iris que es necesaria en estos casos.

Como conclusión, mencionar que en este estudio el síndrome IFIS se presenta en el $67 \%$ de los pacientes en tratamiento con tamsulosina aunque solo el $10 \%$ presenta la tríada completa. En general, estos casos pueden ser intervenidos sin complicaciones por cirujanos expertos y con una depurada técnica quirúrgica.

Por otro lado, y como nuevo hallazgo, cabría destacar la observación de una menor reactividad pupilar posquirúrgica en pacientes tratados con tamsulosina frente a pacientes del grupo control, circunstancia no descrita hasta el momento en la literatura.

\section{BIBLIOGRAFÍA}

1. Vasavada A, Singh R. Phacoemulsification in eyes with a small pupil. J Cataract Refract Surg 2000; 26: 1210-1218.

2. Chang DF, Campbell JR. Intraoperative floppy iris syndrome associated with tamsulosin. J Cataract Refract Surg 2005; 31: 664-673. 
3. Kershner RM. Intraoperative floppy iris syndrome associated with tamsulosin. Letter. J Cataract Refract Surg 2005; 31: 2239.

4. Nguyen $D Q$, Sebastian RT, Kyle G. Intraoperative floppy iris syndrome associated with tamsulosin. Letter. J Cataract Refract Surg 2005; 31: 2240.

5. Nguyen $D Q$, Sebastian RT, Philip J. Intraoperative floppy iris syndrome associated with tamsulosin. Letter. BJU Int 2006; 97: 197-199.

6. Lawrentschuk N, Bylsma G. Intraoperative floppy iris syndrome and its relationship to tamsulosin: a urologist's guide. BJU Int 2006; 97: 2-4.

7. Schwinn DA, Afshari NA. Alphal-adrenergic antagonists and floppy iris syndrome: tip of the iceberg? Ophthalmology 2005; 112: 2059-2060.

8. Wikberg-Matsson A, Uhlen S, Wikberg JE. Characterization of alphal-adrenoceptor subtypes in the eye. Exp Eye Res 2000; 70: 51-60.

9. O'leary MP. Tamsulosin: current clinical experience. Urology 2001; 58: 42-48.
10. Chapple CR. The oral controlled absorption system (OCAS): the evolution of tamsulosin for the treatment of lower urinary tract symptoms suggestive of benign prostatic hyperplasia (LUTS/BPH). Eur Urol Suppl 2005; 4: 20-22.

11. Djavan B. Alpha-1 adrenoceptor antagonists for the treatment of lower urinary tract symptoms suggestive of benign prostatic hyperplasia (LUTS/BPH): state of the art. Eur Urol Suppl 2004; 3: 23-30.

12. Debruyne FM. Alpha blockers: are all created equal? Urology 2000; 56: 20-22.

13. Arshinoff SA. Modified SST-USST for tamsulosin-associated intraocular floppy-iris syndrome. J Cataract Refract Surg 2006; 32: 559-561.

14. Walkow T, Anders N, Klebe S. Endothelial cell loss after phacoemulsification: relation to preoperative and intraoperative parameters. J Cataract Refract Surg 2000; 26: 727-732.

15. Cheung CM, Awan MA, Sandramouli S. Prevalence and clinical findings of tamsulosin-associated intraoperative floppy-iris syndrome. J Cataract Refract Surg 2006; 32: 1336-1339. 